\title{
Output tracking control of nonlinear systems with mismatched uncertainties *
}

\author{
Teh-Lu Liao a , Li-Chen Fu a,b \\ and Chen-Fa Hsu ${ }^{\text {a }}$ \\ "Department of Electrical Engineering \\ ${ }^{b}$ Department of Computer Science and Information Engineer- \\ ing, National Taiwan Unicersity, Taipei, Taiwan, ROC
}

Received 3 November 1990

Revised 15 March and 6 August 1991

\begin{abstract}
This paper addresses the output tracking problem of a class of nonlinear systems. Given input-output linearizability of the class of nonlinear systems, a robust output tracking controller derived via a Lyapunov-based approach is proposed. Here, the model uncertainties do not need to satisfy the conventional matching condition but states and tracking error remain bounded. Furthermore, the magnitude of the tracking error will bear dominant dependence on the magnitude of the mismatched uncertainties.
\end{abstract}

Keywords: Output tracking control; input-output linearizable systems; mismatched uncertainty nonlinear systems.

\section{Introduction}

After the advent of the work of $[4,16]$, the use of differential geometric methods for nonlinear state feedback has received considerable attention in the literature. In almost all the research works, linearization of the closed-loop system is incorporated in various ways, e.g., linearization of the closed-loop state equations $[13,14]$ or linearization of the closed-loop input-output map $[15,19]$. Feedback linearizable systems are especially appealing from the point of view of control design. There exist successful implementations in the design of the control for such systems such as flight dynamics [18] and attitude maneuvers [9]. However, for many real systems, there exist inevitable uncertainties in their constructed mod-

\footnotetext{
* This research was supported by National Science Council under the grants CS-78-0210-D002-27 and NSC-79-0404E002-03.
}

els. Therefore, the design of a robust controller that deals with uncertainties of a system is an important subject for the design of a good and efficient control system.

So far, to deal with uncertain nonlinear systems, three main approaches have been proposed: (1) adaptive control, (2) Lyapunov-based control, (3) variable stucture control. The first one is applied to systems with parametrized uncertainties but the other two then allow nonparametrized uncertainties. Specifically, the Lyapunov-based approach $[1,7,8]$ relies on an explicit construction of a Lyapunov function based on which a state feedback control is synthesized using the bounds on the uncertainties. To achieve the objective of either stabilization or tracking, however, some assumptions were introduced regarding the structure of the uncertainties and are often referred to as matching condition.

In recent years, the robustness analysis of uncertain dynamical systems without the matching condition has been conducted [2,7,20,21]. The work in $[2,20]$ proposes the critical mismatch threshold condition to guarantee overall stability for the class of linear uncertain systems, whereas the work in [21], which mainly deals with a class of nonlinear systems with a linear nominal part and a nonlinear uncertain part, attains the similar stability property. As to the work in [7], it introduces the notion of mismatch envelope as a measure of severeness of mismatch and, then, proposes a practical stability criterion over that measure. Finally, the recent work in [3] brings up the so-called generalized matching condition for a class of nonlinear systems, but fails to provide a discussion on the effect of that condition to the zero dynamics.

In this paper, the main goal is to study the output tracking of a class of nonlinear systems of which the uncertainties may not satisfy the conventional matching condition. The uncertainties 
are generally decomposed into two parts: the matched part which lies in the range space of $g(x)$ and the mismatched part which resides in the null space of $g(x)$. With previous results $[1,8,10,17]$, arbitrarily large matched uncertainties can be compensated for by some properly designed controller. As to the effects of the mismatched uncertainties to the system and to the tracking performance, the paper provides a detailed study and proposes a suitable controller. It can be shown that the tracking error will converge to a small set whose size can be directly related to the magnitude of the mismatched uncertainties.

The robust controller design can be approached in the following steps: (1) under suitable assumptions for the nominal system, a linearizing control law is devised, which intends to linearize the input-output map; (2) after the bounds on the effect of the matched uncertainties to the input-output map is derived, a robust control law is designed to compensate for that effect by a Lyapunov-based approach; (3) the allowable maximum bound on the effect due to mismatched uncertainties is given as robustness margin to guarantee the system stability, whereas the error tracking performance is established in terms of the effect of that mismatched part.

\section{System formulation}

For the simplicity of exposition, we restrict ourselves to consider a single-input single-output nonlinear system in the presence of uncertainties as follows:

$\dot{x}=f(x)+\Delta f(x)+(g(x)+\Delta g(x)) u$,

$y=h(x)$,

where $x \in \mathbb{R}^{n}, u \in \mathbb{R}, y \in \mathbb{R}$, are state variables, input, and output respectively, $f(\cdot), g(\cdot), \Delta f(\cdot)$ and $\Delta g(\cdot)$ are smooth vector fields in $\mathbb{R}^{n}$ with $f(0)=0$, and $h(\cdot)$ is a smooth function satisfying $h(0)=0$. The nominal system is then defined as follows:

$\dot{x}=f(x)+g(x) u$,

$y=h(x)$,

i.e., $\Delta f(x) \equiv 0$ and $\Delta g(x) \equiv 0$ in $(2.1)$
There has been a great deal of excitement in recent years over the development of a rather complete theory for explicitly linearizing the input-output map of the nominal system (2.2) using state feedback. This has been worked out in several papers like those of $[5,15,19]$.

In the following notations $L_{f} h(x)$ and $L_{g} h(x)$ denote, respectively, the Lie derivative of the function $h(x)$ with respect to the vector field $f$ and that with respect to $g$. High order Lie derivatives can be defined recursively such as

$L_{f}^{k} h(x)=L_{f}\left(L_{f}^{k-1} h(x)\right), \quad k>1$.

The notation ad $\operatorname{ad}_{f} g(x)$ denotes the Lie bracket of the vector fields $f$ and $g$, and $\Delta$ denotes the distribution spanned by the vector field $g$. Furthermore, the minimum (resp. maximum) eigenvalue of a matrix is denoted as $\lambda_{\text {min }}(\cdot)$ (resp. $\left.\lambda_{\max }(\cdot)\right)$, whereas the transpose of a vector or of a matrix is written as $(\cdot)^{\mathrm{t}}$ and $\|\cdot\|$ denotes the Euclidean norm.

Definition 2.1 (Class $\mathrm{K}$ function). A function

$\alpha(\cdot, \cdot):, R_{+} \times R_{+} \times R_{+} \rightarrow R_{+}$

belongs to class $\mathrm{K}$, if it is continuous, strictly increasing for each argument, and $\alpha(0,0,0)=0$.

Definition 2.2. (Strong relative degree). The strong relative degree of the output of the system (2.2) is defined as $r$ if

$L_{g} h(x)=L_{g} L_{f} h(x)=\cdots=L_{g} L_{f}^{r-2} h(x) \equiv 0$

and

$L_{g} L_{f}^{r-1} h(x) \neq 0, \quad$ for all $x \in \mathbb{R}^{n}$.

Throughout this paper we will assume that the nominal system (2.2) possesses strong relative degree $r<\infty$. Associated with this assumption, it has been shown [15] that there exists a local coordinate transformation,

$$
\begin{aligned}
&\left(\xi^{\mathrm{t}}, \eta^{\mathrm{t}}\right)^{\dagger}= T(x) \\
& \equiv\left(h(x), L_{f} h(x), \ldots, L_{f}^{r-1} h(x),\right. \\
&\left.T_{1}(x), \ldots, T_{n-r}(x)\right)^{\mathrm{t}}
\end{aligned}
$$

satisfying

$L_{g} T_{i}(x)=0, \quad i=1, \ldots, n-r$, 
such that (2.2) can be transformed into the following normal form:

$\dot{\xi}_{i}=\xi_{i+1}, \quad i=1, \ldots, r-1$,

$\dot{\xi}_{r}=a(\xi, \eta)+b(\xi, \eta) u$

$\dot{\eta}=q(\xi, \eta), \quad \xi \in \mathbb{R}^{r}, \eta \in \mathbb{R}^{n-r}$,

$y=\xi_{1}$,

where $a(\xi, \eta)$ and $b(\xi, \eta)$ stand for $L_{f}^{r} h(x)$ and $L_{g} L_{f}^{r-1} h(x)$ in $(\xi, \eta)$ coordinate, respectively, and $q_{i}(\xi, \eta)$ represents $L_{f} T_{i}(x), i=1, \ldots, n-r$, in $(\xi, \eta)$ coordinate.

Since $b(\xi, \eta)$ is bounded away from zero from the hypothesis on the relative degree of the nominal system, its inverse is well defined and so is linearizing law derived from (2.3), namely,

$$
\begin{aligned}
u & =\left.\frac{1}{b(\xi, \eta)}(-a(\xi, \eta)+w)\right|_{\left(\xi^{\prime}, \eta^{\prime}\right)^{t}=T(x)} \\
& =\frac{1}{b(x)}(-a(x)+w) \\
& \equiv u_{a}
\end{aligned}
$$

which linearizes the input--output map of (2.2). Here, $w$ is a new control to be designed for the purpose of tracking. Note that for clarity sake we have abused the notation by letting

$\left.a(\xi, \eta)\right|_{\left(\xi^{t}, \eta^{\prime}\right)^{t}=T(x)}=a(x)$ and

$\left.b(\xi, \eta)\right|_{\left(\xi^{\prime}, \eta^{\prime}\right)^{\prime}=T(x)}=b(x)$.

The control law (2.4) also makes the state vector $\eta$ completely unobservable from the output. It can be easily verified that the equation

$\dot{\eta}=q(0, \eta)$

characterizes the zero dynamics of the system (2.2).

The previous analysis shows that the normal form (2.3) and the zero dynamics (2.5) are locally defined. For the existence of a globally defined normal form of the type (2.3), necessary and sufficient conditions have been investigated in [6]. In addition to the strong relative degree assumption, a further property on a set of $r$ vector fields is required. It is stated in the following assumption:

(A1) Defines $L_{0}$ as

$$
\begin{aligned}
L_{0}=\left\{x \in \mathbb{R}^{n}: h(x)\right. & =L_{f} h(x)=\cdots \\
& \left.=L_{f}^{r-1} h(x)=0\right\},
\end{aligned}
$$

which is a smooth submanifold of $\mathbb{R}^{n}$. Let $L_{0}$ be connected and the zero dynamics (2.5) be globally asymptotically stable (i.e., the system (1.2) is globally minimum-phase). Moreover, the vector fields

$\bar{g}(x), \operatorname{ad}_{\bar{f}} \bar{g}(x), \ldots, \operatorname{ad}_{\bar{f}}^{r-1} \bar{g}(x)$,

where

$\bar{g}(x)=\frac{1}{L_{g} L_{f}^{r-1} h(x)} g(x)$

and

$\bar{f}(x)=f(x)-\frac{L_{f}^{r} h(x)}{L_{g} L_{f}^{r-1} h(x)} g(x)$,

are complete.

If (A1) is satisfied, then a globally defined diffeomorphism exists and the zero dynamics globally exists as well. But to achieve output tracking, the following assumption will be necessary.

(A2) The equilibrium point, $\eta=0$, of the zero dynamics (2.5) is globally exponentially stable. Moreover, the function $q(\xi, \eta)$ is Lipschitz in $\xi$ uniformly in $\eta$.

Remarks. (1) Since the zero dynamics is exponentially stable by assumption, therefore, by a converse theorem of Lyapunov [11], there exists a Lyapunov function $V_{0}(\eta)$ which satisfies the following inequalities:

$k_{1}\|\eta\|^{2} \leq V_{0}(\eta) \leq k_{2}\|\eta\|^{2}$,

$\frac{\partial V_{0}}{\partial \eta} q(0, \eta) \leq-k_{3}\|\eta\|^{2}$,

$\left\|\frac{\partial V_{0}}{\partial \eta}\right\| \leq k_{4}\|\eta\|$

where $k_{1}, k_{2}, k_{3}$ and $k_{4}$ are some appropriate positive constants.

(2) Since $q(\xi, \eta)$ is Lipschitz in $\xi$, there exists a positive constant $L$ such that

$\|q(\xi, \eta)-q(0, \eta)\| \leq L\|\xi\|$ for all $\eta \in \mathbb{R}^{n-r}$, and $\mathrm{L}$ is called a Lipschitz constant of $q(\xi, \eta)$. 
To date, most of the existing theorems on controlling the class of uncertain nonlinear systems such as the one given in (2.1) require additional assumptions to constrain the structure of the uncertain vector fields $\Delta f(x)$ and $\Delta g(x)$. Under such assumptions, a conventional matching condition is imposed for tasks of stabilization or tracking $[1,8]$. Here, in this paper we will replace this restrictive condition by a much more relaxed one stated in the following assumption.

(A3) There exist smooth functions $\delta_{1}(\cdot), \delta_{2}(\cdot)$ : $\mathbb{R}^{n} \rightarrow \mathbb{R}$ and $f_{1}(\cdot), g_{1}(\cdot): \mathbb{R}^{n} \rightarrow \mathbb{R}^{n}$ such that the uncertainties $\Delta f(x)$ and $\Delta g(x)$ in (2.1), for all $x \in \mathbb{R}^{n}$, satisfy

$\Delta f(x)=g(x) \delta_{1}(x)+f_{1}(x)$,

$\Delta g(x)=g(x) \delta_{2}(x)+g_{1}(x)$,

Moreover, $f_{1}^{t}$, and $g_{1}^{t}$ are in $\Delta^{\perp}$, where $\Delta^{\perp}$ is the annihilator of $\Delta$.

Remark. In words, the assumption decomposes $\Delta f(x)$ and $\Delta g(x)$ respectively into two parts, where $g(x) \delta_{1}(x)$ and $g(x) \delta_{2}(x)$ are in general referred to as the matched uncertainties, which can be compesated for by the controller proposed in $[1,10,16]$, whereas $f_{1}(x)$ and $g_{1}(x)$ can be viewed as the mismatched uncertainties which will degrade the performance of tracking [5]. Clearly, $f_{1}$ and $g_{1}$ are uniquely defined given $\Delta f$ and $\Delta g$.

Another assumption which governs the desired output trajectory $y_{\mathrm{d}}(t)$ is needed here.

(A4) The desired output trajectory $y_{\mathrm{d}}(t)$ and its first $r$ derivatives (i.e., $y_{\mathrm{d}}, y_{\mathrm{d}}^{(1)}, \ldots, y_{\mathrm{d}}^{(r)}$ ) are all uniformly bounded, and satisfy

$\left\|\left(y_{\mathrm{d}}, y_{\mathrm{d}}^{(1)}, \ldots, y_{\mathrm{d}}^{(r)}\right)\right\| \leq b_{\mathrm{d}}$

for some positive constant $b_{\mathrm{d}}$.

Now the objective of the paper can be stated as follows: given the desired trajectory $y_{\mathrm{d}}(t)$, design a control law incorporating the linearizing control (2.4) so that the output error of the closed-loop system will be bounded, and will be related to the effect of mismatched uncertainty while maintaining the boundedness of all signals inside the loop disregarding the uncertainties $\Delta f(x)$ and $\Delta g(x)$.

\section{Robust tracking controller and main result}

Consider the system (2.1) and let the uncertain vector fields $\Delta f(x)$ and $\Delta g(x)$ satisfy assumption (A3). By some derivations, it can be easily verified that (2.1) can be transformed into the following form:

$$
\begin{aligned}
\dot{\xi}_{i} & =\xi_{i+1}+L_{f_{1}} L_{f}^{i-1} h(x)+L_{g_{1}} L_{f}^{i-1} h(x) u \\
& i=1, \ldots, r-1 \\
\dot{\xi_{r}}= & a(\xi, \eta)+b(\xi, \eta)\left(\delta_{1}(x)\right)+\left(1+\delta_{2}(x)\right) u \\
& \quad+L_{f_{1}} L_{f}^{r-1} h(x)+L_{g_{1}} L_{f}^{r-1} h(x) u, \\
\dot{\eta} & =q(\xi, \eta)+\phi(x, u), \\
y & =\xi_{1}
\end{aligned}
$$

where

$$
\begin{gathered}
\phi_{i}(x, u)=L_{f_{1}} T_{i}(x)+L_{g_{1}} T_{i}(x) u, \\
i=1, \ldots, n-r .
\end{gathered}
$$

Define the trajectory error, $e \in \mathbb{R}^{r}$, to be

$$
e=\left[\begin{array}{c}
e_{1} \\
e_{2} \\
\vdots \\
e_{r}
\end{array}\right]=\left[\begin{array}{c}
\xi_{1} \\
\xi_{2} \\
\vdots \\
\xi_{r}
\end{array}\right]-\left[\begin{array}{c}
y_{\mathrm{d}} \\
y_{\mathrm{d}}^{(1)} \\
\vdots \\
y_{\mathrm{d}}^{(r-1)}
\end{array}\right]
$$

and an auxiliary signal $\psi(x, u): \mathbb{R}^{n} \times \mathbb{R} \rightarrow \mathbb{R}^{r}$, as

$\psi(x, u)=\left[\begin{array}{c}L_{f_{\mathrm{t}}} h(x)+L_{g_{1}} h(x) u \\ L_{f_{\mathrm{t}}} L_{f}^{r-1} h(x)+L_{g_{1}} L_{f}^{r-1} h(x) u\end{array}\right]$.

Then, the system (3.1) with the control law (2.4) can be expressed as

$$
\begin{aligned}
{\left[\begin{array}{c}
\dot{e}_{1} \\
\dot{e}_{2} \\
\vdots \\
\dot{e}_{r}
\end{array}\right]=} & {\left[\begin{array}{cccccc}
0 & 1 & 0 & \ldots & \ldots & 0 \\
0 & 0 & 1 & 0 & \ldots & 0 \\
\vdots & \vdots & \vdots & \vdots & \ddots & \vdots \\
0 & 0 & 0 & \ldots & \ldots & 0
\end{array}\right]\left[\begin{array}{c}
e_{1} \\
e_{2} \\
\vdots \\
e_{r}
\end{array}\right] } \\
& +\left[\begin{array}{c}
0 \\
0 \\
\vdots \\
1
\end{array}\right]\left(-y_{\mathrm{d}}^{(r)}+\delta_{1}(x) b(x)\right. \\
& +\psi\left(x, u_{a}\right),
\end{aligned}
$$


$\dot{\eta}=q(\xi, \eta)+\phi\left(x, u_{a}\right)$,

$y=\xi_{1}$,

or in a more compact form as

$$
\begin{aligned}
\dot{e}= & A e+B\left(-y_{\mathrm{d}}^{(r)}+\delta_{1}(x) b(x)\right. \\
& \left.-\delta_{2}(x) a(x)+\left(1+\delta_{2}(x)\right) w\right) \\
& +\psi\left(x, u_{a}\right), \\
\dot{\eta}= & q(\xi, \eta)+\phi\left(x, u_{a}\right) .
\end{aligned}
$$

Let us define

$\bar{e}=-\left(a_{r} e_{1}+\cdots+a_{1} e_{r}\right)$

and

$A_{\mathrm{c}}=\left[\begin{array}{rrrrrr}0 & 1 & 0 & \ldots & \ldots & 0 \\ 0 & 0 & 1 & 0 & \cdots & 0 \\ & & \vdots & & & \\ -a_{r} & \ldots & & \ldots & & -a_{r}\end{array}\right]$,

where the parameters $a_{i}, i=1, \ldots, r$, are chosen in such a way that the matrix is Hurwitz.

In order to reduce the effects of the uncertainty terms to the trajectory error $e$ while maintaining the boundedness of $\eta$, we will choose a large gain in the control $w$ to compensate for the matched uncertainties disregarding the presence of slight mismatched uncertainties. Before this becomes possible, the knowledge of bounds on the uncertainties is required a priori. This is stated in the following assumptions.

(A5) There exist known functions $\beta_{1}(\cdot): \mathbb{R}^{n} \rightarrow \mathbb{R}_{+}$, $\beta_{2}(\cdot): \mathbb{R}^{n} \rightarrow \mathbb{R}_{+}$, such that, for all $x \in \mathbb{R}^{n}$,

$1+\delta_{2}(x) \geq \beta_{1}(x)>0$,

$\left\|\delta_{1}(x) b(x)-\delta_{2}(x) a(x)+\delta_{2}(x)\left(\bar{e}+y_{\mathrm{d}}^{(r)}\right)\right\|$

$$
\leq \beta_{2}(x) \text {. }
$$

(A6) There exist four positive constants $l_{1}, l_{2}, l_{3}$ and $l_{4}$ such that, for all $x \in \mathbb{R}^{n}$,

$$
\left\|\left.P_{\mathrm{c}} \psi\left(x, u_{a}\right)\right|_{x=T^{-1}\left(\xi^{\prime}, \eta^{\prime}\right)}\right\| \leq l_{1}(\|\xi\|+\|\eta\|)+l_{3},
$$

$\left\|\left.\phi\left(x, u_{a}\right)\right|_{x=T^{-1}\left(\xi^{t} \cdot \eta^{\prime}\right)}\right\| \leq l_{2}(\|\xi\|+\|\eta\|)+l_{4}$,

where $P_{\mathrm{c}}$, a positive definite symmetric matrix, is a solution to the Lyapunov equation

$P_{\mathrm{c}} A_{\mathrm{c}}+A_{\mathrm{c}}^{\mathrm{t}} P_{\mathrm{c}}=-2 I$.
Remark. Althought the verification of the assumptions (A5)-(A6) is not trivial, for a given particular system, especially a mechanical system, (A5) turns out to be quite straighforward [10]. If $f_{1}(x)$ and $g_{1}(x)$ are smooth enough, (A6) will be surely satisfied within a compact set $B_{R}$, a ball in $\mathbb{R}^{n}$ with radius $R$. Of course, $R$ can be arbitrarily large and $l_{i}, i=1,2,3,4$, will then depend on $R$.

Now we devise the control signal $w$ to be the following form:

$w=\bar{e}+y_{\mathrm{d}}^{(r)}-\gamma(x) \operatorname{sat}\left(\beta_{2}(x) B^{t} P_{\mathrm{c}} e\right)$

where $\gamma(x)$ satisfies

$\gamma(x) \geq \frac{\beta_{2}(x)}{\beta_{1}(x)}$

and $\operatorname{sat}(\cdot)$ is the saturation function defined as

$\operatorname{sat}(\zeta)= \begin{cases}\frac{\zeta}{|\zeta|} & \text { if }|\zeta| \geq 0, \\ \zeta / \varepsilon & \text { if }|\zeta| \leq \varepsilon,\end{cases}$

for some small positive design parameter $\varepsilon$. With the control law given by (3.8) and (3.9), the equation (3.4) can be rewritten as

$$
\begin{aligned}
\dot{e}= & A_{\mathrm{c}} e+B\left(\delta_{1}(x) b(x)-\delta_{2}(x) a(x)\right. \\
& \left.+\delta_{2}(x)\left(\bar{e}+y_{\mathrm{d}}^{(r)}\right)\right) \\
& -B\left(\left(1+\delta_{2}(x)\right)+\gamma(x) \operatorname{sat}\left(\beta_{2}(x) B^{\mathrm{t}} P_{\mathrm{c}} e\right)\right) \\
& +\psi\left(x, u_{a}\right), \\
\dot{\eta}= & q(\xi, \eta)+\phi\left(x, u_{a}\right) .
\end{aligned}
$$

Subsequently, we will show that such a controller design will tolerate the mismatched uncertainties to some extent to still achieve the overall system stability and some degree of tracking performance. In fact, the magnitude of the ultimate tracking errors will dominantly depend on the magnitude of the mismatched uncertainties since $\varepsilon$ can be made arbitrarily small. All those properties are summarized in the following theorem, which is proved in the Appendix.

Theorem (Global stability and bounded tracking). Consider the system (2.1), (2.4), (3.8) and (3.9) satisfying (A1)-(A3), (A5)-(A6), and the desired output trajectory satisfying the assumption (A4). 
Then there exist $l_{1}^{*}$ and $l_{2}^{*}$ such that, for all $0 \leq l_{1}<\min \left(\frac{1}{8}, l_{1}^{*}\right)$ and $l_{2} \in\left[0, l_{2}^{*}\right)$, the states and the tracking errors of the closed-loop system are uniformly bounded for all initial conditions. Furthermore, the tracking error $\left|e_{1}\right|$ will converge to a ball around 0 whose radius can be written explicitly as a class $\mathrm{K}$ function of $\left(l_{1}, l_{3}, \varepsilon\right)$.

Remarks. (1) Since $\Delta f(x)$ and $\Delta g(x)$ do not satisfy the conventional matching condition, there will exist uncertainty terms in the unobservable dynamics. Here, $l_{2}, l_{4}$ can be considered as a measure of the effect of mismatched uncertainties to the unobservable dynamics. By the same token, $l_{1}, l_{3}$ may be considered as a measure of the effect of mismatched unccrtainties to the tracking error dynamics.

(2) If $f_{1}(x)=0, g_{1}(x)=0$, i.e., $l_{i}=0, i=1,2$, 3,4 , the assumption (A3) is simply the conventional matching condition. In this case, the control law (3.7) can be reduced to a switching control, i.e., having $\varepsilon=0$, and, hence, the asymptotic tracking will be allained [10].

(3) If the assumption (A6) is satisfied as described in the remark after equation (2.7), then after slight modification of the proof in the Appendix it can be shown that the results of the theorem remain valid.

\section{An illustration example}

To illustrate the effectiveness of the controller, consider the following example. Let the nonlinear system be given as

$\dot{x}=f(x)+\Delta f(x)+(g(x)+\Delta g(x)) u$,

$y=h(x)$,

where

$$
\begin{aligned}
& f(x)=\left[\begin{array}{c}
-2 x_{1}+x_{1} x_{2} \\
0
\end{array}\right], \quad g(x)=\left[\begin{array}{l}
0 \\
1
\end{array}\right], \\
& \Delta f(x)=\left[\begin{array}{c}
0.1\left(x_{1}+x_{2}\right) \\
0.1 x_{1} x_{2}
\end{array}\right], \\
& \Delta g(x)=\left[\begin{array}{c}
0.1 \cos \left(x_{1}\right) \\
0.1 \sin \left(x_{2}\right)
\end{array}\right],
\end{aligned}
$$

and $h(x)=x_{2}$. It can be easily verified that

$a(x)=L_{f} h(x)-0$,

$b(x)=L_{g} h(x)=1 \neq 0$

for all $x$ and, thus, the strong relative degree of the nominal system is 1 . From (2.4), we obtain

$$
\begin{aligned}
u & =\frac{1}{L_{g} h(x)}\left(-L_{f} h(x)+w\right) \\
& =w
\end{aligned}
$$

and the zero dynamics can be found to be

$\dot{x}_{1}=-2 x_{1}$

which is exponentially stable. Therefore, the assumption (A2) is satisfied.

We can, then, decompose $\Delta f(x)$ and $\Delta g(x)$ such that

$\delta_{1}(x)=0.1 x_{1} x_{2}, \quad \delta_{2}(x)=0.1 \sin \left(x_{2}\right)$,

and

$f_{1}(x)=\left[\begin{array}{c}0.1\left(x_{1}+x_{2}\right) \\ 0\end{array}\right]$,
$g_{1}(x)=\left[\begin{array}{c}0.1 \cos \left(x_{1}\right) \\ 0\end{array}\right]$.

By a suitable choice, we have $A_{\mathrm{c}}=[-5], \bar{e}=$ $-5\left(y-y_{\mathrm{d}}\right)$ and let the desired output $y_{\mathrm{d}}(t)=$ $\sin (t)$. After solving the Lyapunov equation in (3.7), $P_{\mathrm{c}}$ is obtained as $P_{\mathrm{c}}=[0.2]$. Now we evaluate the assumption in (A5) and find that

$$
\begin{aligned}
& 1+\delta_{2}(x) \geq 0.8+0.1 \sin \left(x_{2}\right)=\beta_{1}(x), \\
& \left\|\delta_{1}(x) b(x)-\delta_{2}(x) a(x)+\delta_{2}(x)\left(\bar{e}+\dot{y}_{\mathrm{d}}\right)\right\| \\
& \quad \leq\left|0.1 x_{1} x_{2}\right|+\left|5\left(y-y_{\mathrm{d}}\right)+1\right|=\beta_{2}(x),
\end{aligned}
$$

so that the feedback gain can be chosen as

$$
\gamma(x)=\beta_{2}(x) / \beta_{1}(x) \text {. }
$$

Finally, the control input $w$ is set as

$$
w=\bar{e}+\dot{y}_{\mathrm{d}}-\gamma(x) \operatorname{sat}\left(0.2 \beta_{2}(x)\left(y-y_{\mathrm{d}}\right)\right)
$$

and, then, is applied to the true system. The trajectorics of the output tracking error $e_{1}$ and one of the state variables, $x_{1}$, are shown in Figure 1 , where $\varepsilon=0.1$. With the same initial condition 
as in the previous case but with $\varepsilon=0.01$, the results are shown in Figure 2. Clearly, the output of the closed-loop system is uniformly ultimately bounded as predicted. Furthermore, the output error $e_{1}$ becomes smaller in the second case than that in the first case.

\section{Conclusions}

In this paper, we have treated the robust tracking problem of a class of uncertain nonlinear systems that do not satisfy the conventional matching condition. We assume that the nominal system is linearizable and minimum phase. Then, a robust controller has been proposed to guarantee the uniform boundedness of signals inside the closed-loop system and of the tracking error. Furthermore, the magnitude of the tracking error will mainly depend on the magnitude of the mismatched uncertainties since the design parameter $\varepsilon$ can always be chosen to be sufficiently small.

\section{Appendix}

Proof of Theorem 1. To obtain the boundedness result, we proceed with the following Lyapunov
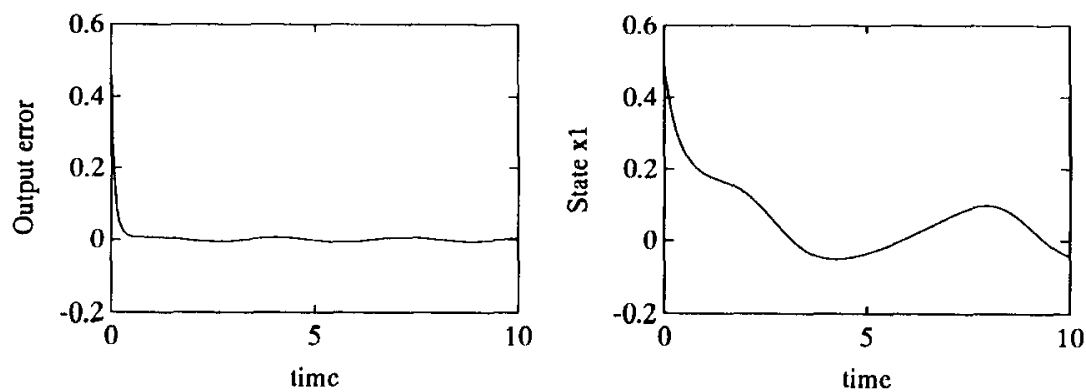

Fig. 1. Output error history and state $x_{1}$ history for $x_{1}(0)=0.5, x_{2}(0)=0.5$ and $\varepsilon=0.1$
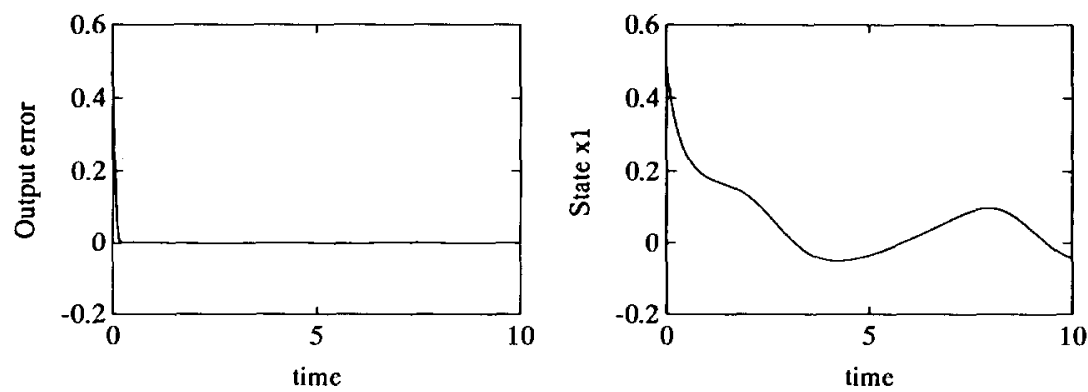

Fig. 2. Output error history and state $x_{1}$ history for $x_{1}(0)=0.5, x_{2}(0)=0.5$ and $\varepsilon=0.01$. 
Similarly, differentiating $V_{0}(\eta)$ along the trajectory of (3.12), we obtain

$$
\begin{aligned}
\dot{V}_{0}(\eta)= & \frac{\partial V_{0}}{\partial \eta}\left(q(\xi, \eta)+\phi\left(x, u_{a}\right)\right) \\
= & \frac{\partial V_{0}}{\partial \eta}(q(0, \eta)+q(\xi, \eta)-q(0, \eta) \\
& \left.+\phi\left(x, u_{a}\right)\right) \\
\leq & -k_{3}\|\eta\|^{2}+k_{4} L\|\xi\|\|\eta\| \\
& +k_{4}\|\eta\|\left(l_{2}(\|\xi\|+\|\eta\|)+l_{4}\right) \\
\leq & -k_{3}\|\eta\|^{2}+k_{4} l_{2}\|\eta\|^{2} \\
& +k_{4}\left(L+l_{2}\right)\|\eta\|\|e\| \\
& +k_{4}\left(b_{\mathrm{d}}\left(L+l_{2}\right)+l_{4}\right)\|\eta\| .
\end{aligned}
$$

Combining (A1) and (A2) will yield

$$
\begin{aligned}
\dot{V} \leq & -\left(\frac{1}{4}-l_{1}\right)\|e\|^{2} \\
& +\left[l_{1}^{2}+\left(\mu k_{4}\left(l_{2}+L\right)\right)^{2}-\mu k_{3}\right. \\
& \left.+\frac{1}{4} \mu k_{3}+\mu k_{4} l_{2}\right]\|\eta\|^{2} \\
& +\left(l_{1} b_{\mathrm{d}}+l_{3}\right)^{2}+\frac{\mu k_{4}^{2}\left(\left(L+l_{2}\right) b_{\mathrm{d}}+l_{4}\right)^{2}}{k_{3}}+\frac{1}{4} \varepsilon .
\end{aligned}
$$

If we set

$$
\begin{aligned}
& \mu=\mu^{*} \equiv \frac{2 k_{3}}{\left(k_{3}+4 k_{4} L\right)^{2}}, \\
& l_{1}^{*} \equiv \frac{k_{3}}{4} \mu^{*} \equiv \frac{k_{3}^{2}}{2\left(k_{3}+4 k_{4} L\right)^{2}},
\end{aligned}
$$

and

$l_{2}^{*} \equiv \frac{k_{3}}{4 k_{4}}$,

then the hypothesis of the theorem will imply the following:

$\dot{V} \leq-\frac{1}{8}\|e\|^{2}-\frac{1}{8} \mu^{*} k_{3}\|\eta\|^{2}+d_{0}$

where

$d_{0}=\left(l_{1} b_{\mathrm{d}}+l_{3}\right)^{2}+\frac{\mu^{*} k_{4}^{2}\left(\left(L+l_{2}\right) b_{\mathrm{d}}+l_{4}\right)^{2}}{k_{3}}+\frac{1}{4} \varepsilon$.
Let

$\alpha_{1}=\min \left(\frac{1}{4 \lambda_{\max }\left(P_{\mathrm{c}}\right)}, \frac{\mu^{*} k_{3}}{8 k_{2}}\right)$.

It then follows that

$\dot{V} \leq-\alpha_{1} V+d_{0}$

which implies that

$V(t) \leq\left(V(0)-\frac{d_{0}}{\alpha_{1}}\right) \mathrm{e}^{-\alpha_{1} t}+\frac{d_{0}}{\alpha_{1}}$.

Consequently, the states $e, \eta$ are uniformly bounded for all $t \geq 0$ and will converge to the ball $B(e, \eta)$ given by

$B(e, \eta)=\left\{(e, \eta): V(e, \eta) \leq d_{0} / \alpha_{1}\right\}$.

Suppose $\alpha_{2}>0$ exists such that

$\|\eta\|^{2} \leq \alpha_{2}$.

Then it is clear that $\alpha_{2}$ will depend on $d_{0}, \alpha_{1}$, $\lambda_{\text {min }}\left(P_{\mathrm{c}}\right), \mu^{*}$, and $k_{2}$ but is independent of $l_{1}$.

Now, to obtain the ultimate bound of the tracking errors in terms of the effective magnitude of the mismatched uncertainties (namely, $l_{1}$, $l_{2}, l_{3}$ and $l_{4}$ ), further investigation of (A2) will be needed, i.e., to rewrite (A2) using (A8) as

$$
\begin{aligned}
\dot{V}_{1} \leq & -\|e\|^{2}+\|e\|\left(l_{1}\left(\|e\|+\|\eta\|+b_{\mathrm{d}}\right)+l_{3}\right) \\
& +\frac{1}{4} \varepsilon \\
\leq & -\left(1-\frac{1}{4}-l_{1}-\frac{1}{4}\right)\|e\|^{2}+l_{1}^{2} \alpha_{2}+\left(l_{1} b_{\mathrm{d}}+l_{3}\right)^{2} \\
& +\frac{1}{4} \varepsilon \\
\leq & -\left(\frac{1}{2}-l_{1}\right)\|e\|^{2}+d_{1} \\
\leq & -\alpha_{4} V_{1}+d_{1}
\end{aligned}
$$

where

$\alpha_{4}=\frac{3}{4 \lambda_{\max }\left(P_{\mathrm{c}}\right)}$

and

$d_{1}=l_{1}^{2} \alpha_{2}+\left(l_{1} b_{\mathrm{d}}+l_{3}\right)^{2}+\frac{1}{4} \varepsilon$,

which implies that $e$ will converge to the ball

$B(e)=\left\{e:\|e\| \leq \sqrt{\frac{2 d_{1}}{\lambda_{\min }\left(P_{\mathrm{c}}\right) \alpha_{4}}}\right\}$ 
and, hence,

$\left\|e_{1}\right\| \leq \sqrt{\frac{2 d_{1}}{\lambda_{\min }\left(P_{\mathrm{c}}\right) \alpha_{4}}}$.

Clearly, the radius of the ball can be shown to be a class $\mathrm{K}$ function of $l_{1}, l_{3}$ and $\varepsilon$. This completes the proof.

\section{Acknowledgement}

The authors gratefully acknowledge Dr. J. Hauser for a helpful discussion on the technical proof of this paper.

\section{References}

[1] B.R. Barmish, M. Corless, and G. Leitmann, A new class of stabilization controller for uncertain dynamical system, SIAM J. Control. Optim. 21 (1983) 246-255.

[2] B.R. Barmish, and G. Leitmann, On ultimate boundedness control of uncertain systems in the absence of matching condition, IEEE Trans. Automat. Control 27 (1982) 153-158.

[3] S. Behtash, Robust output tracking for nonlinear systems, Internat. J. Control 51 (1990) 1381-1407.

[4] R.W. Brocket, Feedback invariants for nonlinear systems, IFAC Congress, Helsinki (1978) 1115-1120.

[5] C.I. Byrnes and A. Isidori, Local stabilization of minimum phase nonlinear systems, Systems Control Lett. 10 (1987) 9-17.

[6] C.I. Byrnes and A. Isidori, Asymptotic stabilization of minimum phase nonlinear system, IEEE Trans. Automat. Control (1991), to appear.

[7] Y.H. Chen, and G. Leitmann, Robustness of uncertain system in the absence of matching assumptions, Internat. J. Control 45 (1987) 1527-1544.

[8] M. Corless, and G. Leitmann, Continuous state feedback guaranteeing uniform ultimate boundedness for uncertain dynamical systems, IEEE Trans. Automat. Control 32 (1987) 763-771.

[9] T.A. Dwyer, Exact nonlinear control of spacecraft slewing maneuvers with internal momentum transfer, Proc. of the ALAA Dynamics Specialists Conference (1984).

[10] L.C. Fu and T.L. Liao, Globally stable tracking of nonlinear systems using variable structure control and with an application to a robotic manipulator, IEEE Trans. Automat. Control 35 (1990) 1345-1351.

[11] W. Hahn, Stability of Motion (Springer-Verlag, Berlin 1967).

[12] J. Hauser, S.S. Sastry, and G. Meyer, Nonlinear controller disign for flight control systems, Memo. No. UCB/ ERL M88 / 76, University of California (1988).

[13] L.R. Hunt, R. Su, and G. Meyer, Global transformation of nonlinear systems, IEEE Trans. Automat. Control 28 (1983) 24-31.

[14] L.R. Hunt, R. Su, and G. Meyer, Design for multi-input nonlinear systems, in: R.W. Brockett, R.S. Millman, and H.J. Sussman, Eds., Differential Geometric Control Theory (Brikhauser, Boston, 1983)

[15] A. Isidori, Nonlinear Control Systems (Springer-Verlag, New York, 1989).

[16] A.J. Krener, On the equivalence of control systems and linearization on nonlinear systems, SIAM J. Control Optim. 11 (1973) 670-698.

[17] T.L. Liao, T.L., L.C. Fu and C.F. Hsu, Adaptive robust tracking of nonlinear systems and with application to a robotic manipulator, Systems Control Lett. 15 (1990) 339348.

[18] G. Meyer, The design of exact nonlinear model follows, Proc. of Joint Automatic Control Conference (1981).

[19] S.S. Sastry and A. Isidori, Adaptive control of nonlinear systems, IEEE Trans. Automat. Control 34 (1989) 11231131.

[20] S.N. Singh and A.R. Coelen, Nonlinear control of mismatched uncertain linear system and application to control of aircraft, ASME J. Dynamic System and Control 106 (1984) 203-210.

[21] S.H. Zak, On the stabilization and observation of nonlinear uncertain dynamic systems, IEEE Trans. Automat. Control 35 (1990) 604-707. 